\title{
Impact of implicit effects on uncertainties and sensitivities of the Doppler coefficient of a LWR pin cell
}

\author{
Mathieu Hursin ${ }^{1, a}$, Olivier Leray ${ }^{1}$, Gregory Perret ${ }^{1}$, Andreas Pautz ${ }^{1}$, Friederike Bostelmann ${ }^{2}$, Alexander Aures ${ }^{2}$, \\ and Winfried Zwermann ${ }^{2}$ \\ 1 Paul Scherrer Institut, 5232 Villigen PSI, Switzerland \\ ${ }^{2}$ Gesellschaft für Anlagen- und Reaktorsicherheit (GRS) gGmbH, Boltzmannstr. 14, 85748 Garching, Germany
}

\begin{abstract}
In the present work, PSI and GRS sensitivity analysis (SA) and uncertainty quantification (UQ) methods, SHARK-X and XSUSA respectively, are compared for reactivity coefficient calculation; for reference the results of the TSUNAMI and SAMPLER modules of the SCALE code package are also provided. The main objective of paper is to assess the impact of the implicit effect, e.g., considering the effect of cross section perturbation on the self-shielding calculation, on the Doppler coefficient SA and UQ. Analyses are done for a Light Water Reactor (LWR) pin cell based on Phase I of the UAM LWR benchmark. The negligence of implicit effects in XSUSA and TSUNAMI leads to deviations of a few percent between the sensitivity profiles compared to SAMPLER and TSUNAMI (incl. implicit effects) except for ${ }^{238} \mathrm{U}$ elastic scattering. The implicit effect is much larger for the SHARK-X calculations because of its coarser energy group structure between $10 \mathrm{eV}$ and $10 \mathrm{keV}$ compared to the applied SCALE libraries. It is concluded that the influence of the implicit effect strongly depends on the energy mesh of the nuclear data library of the neutron transport solver involved in the UQ calculations and may be magnified by the response considered.
\end{abstract}

\section{Introduction}

One major part of input uncertainty in nuclear safety analyses is caused by uncertainties of the nuclear data. In established uncertainty evaluation methods, these input uncertainties are propagated through the calculation chain to uncertainties of output quantities. During a transient event, the negative fuel temperature feedback (Doppler coefficient) plays an important role for the reactor safety. For this reason, an accurate quantification of its uncertainty is an essential prerequisite for a safe reactor design. In the present work, PSI and GRS sensitivity analysis (SA) and uncertainty quantification (UQ) methods, SHARK-X $[1,3]$ and XSUSA [4,5] respectively, are compared for Doppler coefficient calculation. For further comparisons, the TSUNAMI and SAMPLER modules of the SCALE6.2 code package [6] are used. Analyses are done for a Light Water Reactor (LWR) pin cell based on Phase I of the UAM LWR benchmark [7]. The Doppler coefficient is determined by investigating the multiplication factor at a fuel temperature of $900 \mathrm{~K}$ and $1500 \mathrm{~K}$ as shown in Eq. (2).

$$
\rho=\left(\frac{1}{k_{\mathrm{inf}}^{900}}-\frac{1}{k_{\mathrm{inf}}^{1500}}\right) / \Delta \mathrm{T}
$$

The sensitivity coefficients can be expressed as the sum of explicit and implicit components [8]. Explicit terms correspond to parameters appearing explicitly in the multigroup neutron-balance equation and can be evaluated using conventional UQ methods; implicit terms correspond to perturbations in the resonance self-shielded cross sections. Some previous studies on the subject $[8,9]$ yielded a

a e-mail: mathieu.hursin@psi.ch significant effect, notably when the considered response is highly sensitive to capture in fertile isotopes such as the Doppler reactivity coefficient [10]. By contrast, others resulted in a negligible effect in terms of uncertainty [4]. The focus of the present work is to assess the impact of the implicit effect on the Doppler coefficient UQ and SA.

\section{Model and simulation tools used for UQ/SA analysis}

\subsection{Model}

The specification of the pin cell used for the uncertainty analyses is based on the TMI-1 PWR unit cell configuration of the OECD/NEA Uncertainty Analysis in Modelling (UAM) benchmark, Exercise I- $1 \mathrm{~b}$.

The fuel pellet diameter is $9.391 \mathrm{~mm}$, the outer diameter of the gap is $9.582 \mathrm{~mm}$ and the outer diameter of the cladding is $10.928 \mathrm{~mm}$. The unit cell pitch is $14.427 \mathrm{~mm}$. The fuel is $\mathrm{UO}_{2}$ with a ${ }^{235} \mathrm{U}$ enrichment of 4.85 wt. $-\%$ and a mass density of $10.2083 \mathrm{~g} / \mathrm{cm}^{3}$. The gap is filled with helium and the cladding consists of Zircaloy4 with a mass density of $6.55 \mathrm{~g} / \mathrm{cm}^{3}$. The moderator density is $0.7484 \mathrm{~g} / \mathrm{cm}^{3}$.

\subsection{Simulation tools}

The methodologies involved in this work to perform UQ and SA are detailed below. Cross section and covariance data used for the analyses with the different tools are given in Table 1.

(c) The Authors, published by EDP Sciences. This is an Open Access article distributed under the terms of the Creative Commons Attribution License 4.0 (http://creativecommons.org/licenses/by/4.0/). 
Table 1. Analysis tools, cross section and covariance data.

\begin{tabular}{|l|l|l|}
\hline Analysis tool & Cross sections & VCM \\
\hline SHARK-X & $586 \mathrm{~g}$ ENDF/B-VII.0 & \\
\cline { 1 - 2 } $\begin{array}{l}\text { XSUSA } \\
\text { (SCALE 6.1) }\end{array}$ & $238 \mathrm{~g}$ ENDF/B-VII.0 & \multirow{2}{*}{ 44 group SCALE 6.1 } \\
\cline { 1 - 2 } $\begin{array}{l}\text { SAMPLER } \\
\text { (SCALE 6.2) }\end{array}$ & 238g ENDF/B-VII.0 & \\
\cline { 1 - 2 } TSUNAMI & $238 \mathrm{~g}$ ENDF/B-VII.0 & \\
(SCALE 6.2) & & \\
\hline
\end{tabular}

\subsubsection{XSUSA}

The GRS code XSUSA (Cross Section Uncertainty and Sensitivity Analysis) is based on the stochastic sampling method for UQ analysis. However, in the present work, a direct perturbation (DP) approach is used to determine the sensitivity coefficients of the Doppler coefficient: an input cross section of interest is sequentially perturbed in each energy group by $\pm 5 \%$. Two transport calculations are then needed to compute the Doppler coefficient as expressed in Eq. (2). The sensitivity coefficient $S_{i}$ is computed as shown in Eq. (2.2.2).

$$
S_{i}=\frac{1}{\rho} \frac{\rho^{P_{i}}-\rho}{0.05}
$$

where $\rho$ and $\rho^{P}$ are the nominal and perturbed Doppler coefficient, respectively. The cross sections are perturbed after the self-shielding calculation. The implicit effects are therefore not taken into account.

\subsubsection{SHARK-X}

The PSI code SHARK-X is a set of Perl-based scripts build around the lattice code CASMO-5 in order to perform uncertainty quantification. The perturbed cross sections are directly introduced in the CASMO-5 code through an in-house routine added to the code. In SHARK$X$, the calculation of Doppler coefficient sensitivity coefficients is based on k-sensitivity coefficients using the Equivalent Generalized Perturbation Theory (EGPT). [12]. The sensitivity coefficient of the Doppler coefficient can be written as

$$
S_{\rho, \alpha}=\frac{1}{|\rho|}\left(S_{k, \alpha}^{\text {pert }} / k_{\mathrm{inf}}^{\text {pert }}-S_{k, \alpha}^{r e f} / k_{\mathrm{inf}}^{r e f}\right)
$$

where $S_{k, \alpha}^{\text {ref }}$ and $S_{k, \alpha}^{\text {pert }}$ are the k-sensitivity coefficients of the reference $(900 \mathrm{~K})$ and perturbed $(1500 \mathrm{~K})$ states.

The CASMO-5 nuclear data library is based on ENDF/B-VII.0 and consists of a 586-group structure with 128 fast groups, 41 resonance groups between $10 \mathrm{eV}$ and $10 \mathrm{keV}, 375$ narrow groups in the low eV range, and 42 thermal groups below $0.625 \mathrm{eV}$. The finest possible energy group structure (586 energy groups) is used to perform the 2D calculation.

By default, the perturbation of the cross sections is done after the self-shielding. Thus, the implicit effect is usually not taken into account [13]. However, a method has been recently developed and implemented in SHARK$\mathrm{X}$ to properly take into account the perturbation of the ${ }^{238}$ Ucapture in the resonance self-shielding calculation of CASMO-5. It is established on the predetermination of effective perturbation factors based on reference and perturbed NJOY calculations using point wise cross section data. The effective perturbation factors are parametrized with respect to perturbation magnitude, the dilution cross section and energy group index. This treatment is currently limited to ${ }^{238} \mathrm{U}$ capture.

\subsubsection{SCALE 6.2}

The SA and UQ modules of SCALE used in the present work are TSUNAMI and SAMPLER of SCALE 6.2.

TSUNAMI (using the two dimensional transport code NEWT) is based on first order perturbation theory. It uses the EGPT approach (TSAR module) to perform the Doppler coefficient SA and UQ. It includes an approximate treatment of the self-shielding perturbation [8]. With SAMPLER, a sequence of SCALE 6.2, DP calculations are performed similar to XSUSA. In contrast to the XSUSA calculations, implicit effects are taken into account by SAMPLER.

For reasons of consistency with the XSUSA calculations, TSUNAMI and SAMPLER were applied in combination with ENDF/B-VII.0 cross section data. The microscopic cross section library consists of 238 energy groups, 74 of them between $10 \mathrm{eV}$ and $10 \mathrm{keV}$. The energy mesh in the resolved resonance range is much finer than in CASMO-5 above $10 \mathrm{eV}$ but much coarser below.

\subsection{Input uncertainty}

For direct perturbation calculations with SHARK-X, XSUSA and SAMPLER, a set of nuclide-reaction pairs were selected based on the main contributors to the Doppler coefficient uncertainty [10], namely ${ }^{238} \mathrm{U}$ capture, elastic and inelastic scattering as well as ${ }^{1} \mathrm{H}$ elastic scattering. The Variance Covariance Matrix (VCM) used in all calculations is the SCALE 6.1 VCM library. The correlation between isotopes and reactions, even though they are available, are neglected in this work for evaluating uncertainties.

\section{Results}

\subsection{Mean values}

The comparison of XSUSA, SHARK-X, SAMPLER and TSUNAMI Doppler coefficient mean values are shown in Table 2. For comparison, the $\mathrm{k}_{\text {inf }}$ of the unperturbed reference state $(900 \mathrm{~K})$ is also given. The mean value of the Doppler coefficient predicted by the TSUNAMI, SAMPLER and SHARK-X are in good agreement while the one predicted by XSUSA differs by about $15 \%$. This appears to be due to improvements in the self-shielding calculations in SCALE 6.2 as compared to SCALE 6.1.

\subsection{Uncertainty quantification}

The relative standard deviation of the Doppler coefficient due to the uncertain nuclear data input is summarized in Table 1 as well as the contribution of each investigated nuclide-reaction pair. The uncertainty in terms of the Doppler coefficient due to nuclear data is in the $1 \%$ range which is consistent with other uncertainty estimates of the literature [14] and covers the mean value differences between TSUNAMI, SAMPLER and SHARK-X.

The major contributor to the uncertainty of the Doppler coefficient is ${ }^{238} \mathrm{U}$ capture, followed by ${ }^{238} \mathrm{U}$ 
Table 2. Doppler coefficient and associated uncertainty from TSUNAMI, SAMPLER, XSUSA and SHARK-X. ("Explicit" refers to results where only the explicit component of the sensitivity coefficient is considered, whereas "Total" refers to results where both explicit and implicit components are considered.)

\begin{tabular}{|c|c|c|c|c|c|c|c|}
\hline \multirow{2}{*}{\multicolumn{2}{|c|}{ Code }} & XSUSA & SAMPLER & \multicolumn{2}{|c|}{ TSUNAMI } & \multicolumn{2}{|c|}{ SHARK-X } \\
\hline & & Explicit & Total & Explicit & Total & Explicit & Total \\
\hline \multicolumn{2}{|c|}{$\mathbf{k}_{\text {inf }}$} & 1.41230 & 1.41406 & \multicolumn{2}{|c|}{1.41171} & \multicolumn{2}{|c|}{1.41593} \\
\hline \multicolumn{2}{|c|}{$\rho(\mathbf{p c m} / \mathbf{K})$} & -1.80 & -1.56 & \multicolumn{2}{|c|}{-1.57} & \multicolumn{2}{|c|}{-1.55} \\
\hline \multirow{5}{*}{ Rel. Std (\%) } & Cumulative $^{a}$ & 1.05 & 1.15 & 1.09 & 1.09 & 1.51 & - \\
\hline & ${ }^{238} \mathrm{U} \sigma_{\mathrm{c}}$ & 0.91 & 0.96 & 0.98 & 0.98 & 1.46 & 0.90 \\
\hline & ${ }^{238} \mathrm{U} \sigma_{\mathrm{s}, \mathrm{el}}$ & 0.29 & 0.45 & 0.19 & 0.24 & 0.01 & - \\
\hline & ${ }^{238} \mathrm{U} \sigma_{\mathrm{s}, \text { inel }}$ & 0.42 & 0.42 & 0.41 & 0.41 & 0.38 & - \\
\hline & ${ }^{\mathrm{I}} \mathrm{H} \sigma_{\mathrm{s}, \mathrm{el}}$ & 0.13 & 0.13 & 0.13 & 0.13 & 0.08 & - \\
\hline
\end{tabular}

a "Cumulative" refers to the cumulated uncertainty of the listed nuclide reactions.

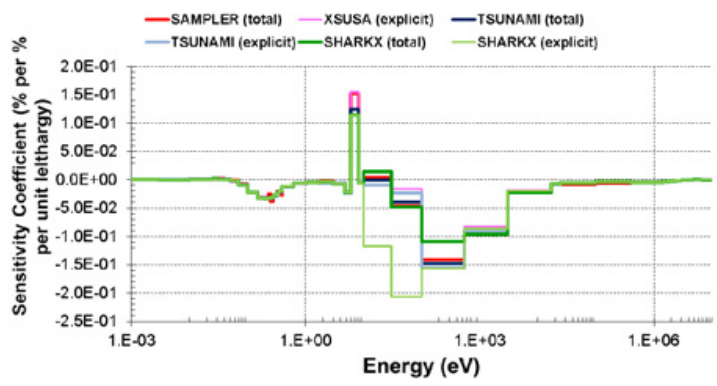

a) ${ }^{238} U$ capture -44 groups

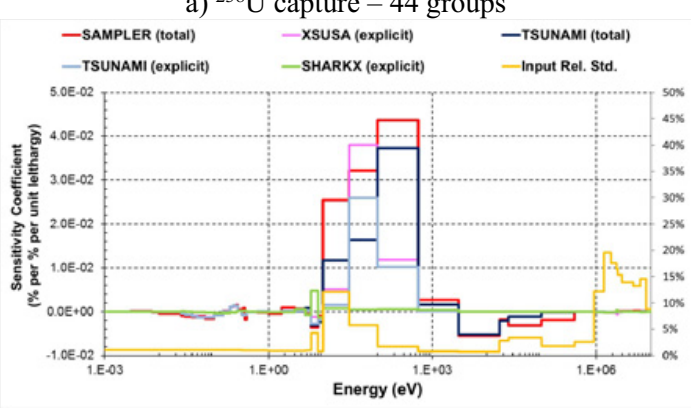

c) ${ }^{238} \mathrm{U}$ elastic scattering -44 groups

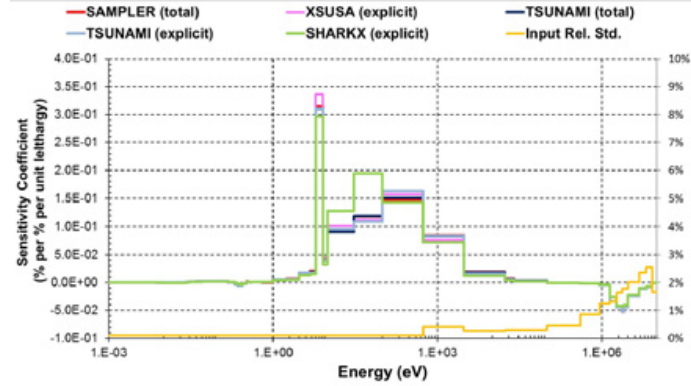

b) ${ }^{1} \mathrm{H}$ inelastic scattering -44 groups

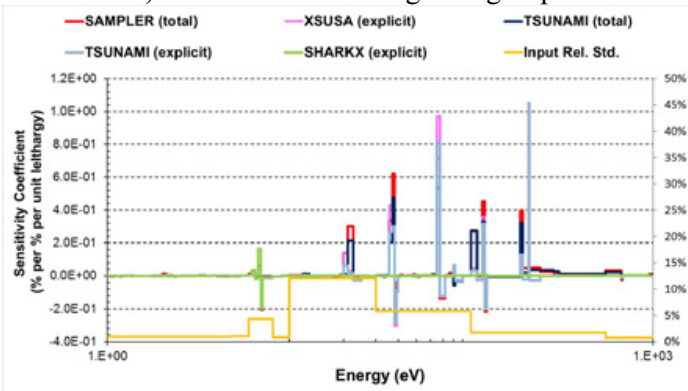

d) ${ }^{238} \mathrm{U}$ elastic scattering -238 groups

Figure 1. Doppler coefficient sensitivity profiles (\%/\%/unit lethargy) for XSUSA, SHARK-X, SAMPLER and TSUNAMI.

inelastic scattering. The nuclide reaction pairs considered in XSUSA, SAMPLER and SHARK-X account for most of the Doppler coefficient uncertainty.

XSUSA, even though it relies on SCALE 6.1 nuclear data and methods as well as it neglects the implicit effect, shows a very good agreement in terms of UQ estimates with TSUNAMI and SAMPLER. In fact, the main differences between the XSUSA and TSUNAMI uncertainties (for ${ }^{238} \mathrm{U}$ capture and elastic scattering) again appear to be due to the changes in the selfshielding calculations from SCALE 6.1 to SCALE 6.2; when using the SCALE 6.1 version of TSUNAMI, the discrepancies to XSUSA are much smaller. SHARK-X UQ estimates considering the implicit effect are in good agreement with TSUNAMI as well. The contribution of the implicit effect to ${ }^{238} \mathrm{U}$ capture is very large in SHARK$\mathrm{X}, 1.46 \%$ considering the explicit effect only, $0.9 \%$ when considering both. For TSUNAMI, however, the differences in terms of Doppler UQ due to the implicit effect are primarily visible on ${ }^{238} \mathrm{U}$ elastic scattering with only minor changes in terms of ${ }^{238} \mathrm{U}$ capture. Since all UQ results rely on the same VCM, the differences observed between the contributions of the various nuclide reaction pairs with SHARK-X compared to the other codes are due to sensitivity coefficients discrepancies.

\subsection{Sensitivity analysis}

The sensitivity coefficients predicted by XSUSA, SAMPLER, TSUNAMI and SHARK-X are shown in Fig. 1: for ${ }^{238} \mathrm{U}$ capture (a) and elastic scattering (c, d), and for ${ }^{1} \mathrm{H}$ elastic scattering (b). Overall, the agreement between the various codes is good except for the ${ }^{238} \mathrm{U}$ elastic scattering. Focusing on this nuclide reaction pair, the major differences between XSUSA and TSUNAMI explicit are located between $10 \mathrm{eV}$ and $1 \mathrm{keV}$. Looking at the more detailed $238 \mathrm{~g}$ sensitivity profiles (4d), the differences appear in the resonances of ${ }^{238} \mathrm{U}$ and can be very large; the reason for the large difference is probably due to the different self-shielding methods between SCALE 6.1 and SCALE 6.2. The large difference in the magnitude of the sensitivity profiles in a region of large input uncertainty (up to $10 \%$ ) explains the UQ discrepancy $(0.3 \%$ for XSUSA vs. $0.2 \%$ for TSUNAMI explicit) observed in Table 2. Considering the SAMPLER and TSUNAMI total sensitivity profiles, their shapes are similar but 
their magnitude is different and may be due to the DP approach used in XSUSA. Finally the SHARK-X explicit and TSUNAMI total sensitivity profiles are similar except between $10 \mathrm{eV}$ to $10 \mathrm{keV}$ where the SHARK$\mathrm{X}$ sensitivities are negligible. The energy range of the discrepancies suggests that they may be due to neglecting the implicit effect in SHARK-X, but since XSUSA and TSUNAMI explicit profiles show non negligible sensitivities in this energy range, the discrepancies may also come from the coarser CASMO-5 energy group structure.

Looking at the implicit effect on the ${ }^{238} \mathrm{U}$ capture sensitivity, it appears to be small in TSUNAMI and much larger in SHARK-X, especially in the $10 \mathrm{eV}$ to $10 \mathrm{keV}$ energy range. When neglecting the implicit effect, the sensitivity magnitude tends to be over-estimated since the effect of the cross section perturbation on the energy self-shielding is neglected: increasing the capture cross section by $20 \%$ will result in an "effective" perturbation of about $10 \%$ due to the increased energy self-shielding. This effective perturbation is only captured if the pointwise flux change due to the increased ${ }^{238} \mathrm{U}$ capture cross section is modelled. Thanks to the fine energy mesh of CASMO-5 in the first resonance of ${ }^{238} \mathrm{U}(6.6 \mathrm{eV})$, even without special treatment of the implicit effect, SHARK$\mathrm{X}$ captures correctly the perturbation of the self-shielding and returns equivalent sensitivities to TSUNAMI total.

However, in the resonance groups of the CASMO-5 library, spanning from $10 \mathrm{eV}$ to $10 \mathrm{keV}$, the magnitude of the sensitivity coefficients calculated by SHARK-X explicit is over-predicted compared to the TSUNAMI one. When considering a treatment of the implicit effect, both TSUNAMI and SHARK-X manage to capture the perturbation of the self-shielding and return consistent sensitivity profiles for ${ }^{238} \mathrm{U}$ capture, which tends to indicate that the implicit effect is significant in the case of SHARK$\mathrm{X}$ and almost negligible in case of TSUNAMI. This behavior is due to the finer energy mesh of TSUNAMI between $10 \mathrm{eV}$ and $10 \mathrm{keV}$, i.e. 74 groups against 41 for CASMO-5. The change of energy self-shielding due to a perturbation in ${ }^{238} \mathrm{U}$ capture cross section is small in TSUNAMI due to its fine energy group structure. It is not the case in SHARK-X. Nonetheless, it appears that even with TSUNAMI, large changes in the magnitude and shape of the sensitivity profile of the Doppler coefficient to ${ }^{238} \mathrm{U}$ elastic scattering can be attributed to the implicit effect. Due to its small input uncertainty, however, the impact in terms of UQ is negligible.

\section{Conclusion}

In the present work, SHARK-X, XSUSA, TSUNAMI and SAMPLER were compared for reactivity coefficient calculations applied to a Light Water Reactor (LWR) pin cell based model. The uncertainty in terms of Doppler coefficient due to nuclear data is in the $1 \%$ range and covers the mean value differences between TSUNAMI, SAMPLER and SHARK-X. When considering the implicit effect, SHARK-X produces Doppler coefficient UQ estimates consistent with TSUNAMI and SAMPLER.

The negligence of implicit effects in XSUSA and TSUNAMI mostly leads to small deviations between the sensitivity profiles compared to SAMPLER and TSUNAMI (incl. implicit effects). It is substantially larger for ${ }^{238} \mathrm{U}$ elastic scattering. The implicit effect in the SHARK-X calculations is very large because of its coarser energy group structure between $10 \mathrm{eV}$ and $10 \mathrm{keV}$ compared to the applied SCALE libraries. It is concluded that the influence of the implicit effect strongly depends on the energy mesh of the nuclear data library of the neutron transport solver involved in the UQ calculations and may be magnified by the response considered.

The work of GRS is supported by the German Federal Ministry for Economic Affairs and Energy.

\section{References}

[1] W. Wieselquist, T. Zhu, A. Vasiliev, and H. Ferroukhi, "PSI Methodologies for Nuclear Data Uncertainty Propagation with CASMO-5M and MCNPX: Results for OECD/NEA UAM Benchmark Phase I," Sci. Technol. Nucl. Install. 15 (2013)

[2] H. Ferroukhi, O. Leray, M. Hursin, A. Vasiliev, G. Perret, and A. Pautz, "Study of Nuclear Decay Data Contribution to Uncertainties in Heat Load Estimations for Spent Fuel Pools," Nucl. Data Sheets 118(1), 498-501 (2014)

[3] M. Hursin, G. Perret, and A. Pautz, "Verification of the new implementations in SHARKX against TSUNAMI to perform pinpower UQ and representativity analysis," Ann. Nucl. Energy 77, 300-309 (2015)

[4] F. Bostelmann, F.-P. Weiss, A. Aures, K. Velkov, W. Zwermann, B.T. Rearden, M.A. Jessee, M.L. Williams, D. Wiarda, and W. Wieselquist, "Uncertainty and Sensitivity Analysis in Criticality Calculations with Perturbation Theory and Sampling," in Proceedings of ANS MC2015

[5] W. Zwermann, B. Krzykacz-Hausmann, L. Gallner, and A. Pautz, "Influence of Nuclear Covariance Data on Reactor Core Calculations," in WONDER 2009

[6] B.T. Rearden, "Overview of SCALE 6.2," in Proceedings of ANS NCSD, Wilmington, NC, 2009

[7] K. Ivanov, M. Avramova, S. Kamerow, I. Kodeli, E. Sartori, E. Ivanov, and O. Cabellos, "Benchmark for Uncertainty Analysis in Modelling (UAM) for Design, Operation and Safety Analysis of LWRs, Volume I: Specification and Support Data for the Neutronics Cases (Phase I)," Report, no. Version 2.1, NEA/NSC/DOC(2012)10, 2016

[8] M.L. Williams, B.L. Broadhead, and C.V Parks, "Eigenvalue Sensitivity Theory for ResonanceShielded Cross Sections," Nucl. Sci. Eng. 177-191 (2001)

[9] B. Foad and T. Takeda, "Importance of selfshielding for improving sensitivity coefficients in light water nuclear reactors," Ann. Nucl. Energy 63, 417-426 (2014)

[10] A. Aures, F. Bostelmann, M. Hursin, and O. Leray, "Benchmarking and Application of the State-ofthe-Art Uncertainty Analysis Methods XSUSA and SHARK-X," Ann. Nucl. Energy, under review, 2016

[11] "SCALE: A Comprehensive Modeling and Simulation Suite for Nuclear Safety Analysis and Design." Oak Ridge, TN. Available from Radiation Safety Information Computational Center at Oak Ridge National Laboratory as CCC-785, 2011 
[12] A. Gandini, G. Palmiotti, and M. Salvatores, "Equivalent generalized perturbation theory (EGPT)," Ann. Nucl. Energy 13(3), 109-114 (1986)

[13] M. Hursin, D. J. Siefman, G. Perret, P. Grimm, and A. Pautz, "Verification of the new implementations in SHARK-X for reactivity coefficients and relative reactivity worth UQ," in PHYSOR 2016 - Unifying Theory and Experiments in the 21st Century, 2016

[14] A. Santamarina and D. Bernard, "Biases and Uncertainties in Doppler reactivity worth calculations," in UAM-9 Meeting, 2015 